\title{
BMJ Open Evaluating the longitudinal risk of social vigilance on atherosclerosis: study protocol for the North Texas Heart Study
}

\author{
John M Ruiz, ${ }^{1}$ Daniel J Taylor, ${ }^{2}$ Bert N Uchino, ${ }^{3}$ Timothy W Smith, ${ }^{3}$ \\ Matthew Allison, ${ }^{4}$ Chul Ahn, ${ }^{5}$ Jillian J Johnson, ${ }^{6}$ Joshua M Smyth ${ }^{6}$
}

To cite: Ruiz JM, Taylor DJ, Uchino BN, et al. Evaluating the longitudinal risk of social vigilance on atherosclerosis: study protocol for the North Texas Heart Study. BMJ Open 2017;7:e017345. doi:10.1136/ bmjopen-2017-017345

- Prepublication history for this paper is available online. To view these files please visit the journal online (http://dx.doi org/10.1136/bmjopen-2017017345).

Received 19 April 2017 Accepted 24 April 2017

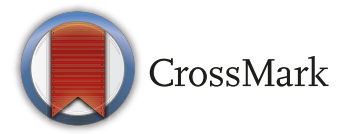

${ }^{1}$ Department of Psychology, University of Arizona, Tucson, Arizona, USA

${ }^{2}$ Department of Psychology, University of North Texas, Denton, Texas, USA ${ }^{3}$ Department of Psychology, University of Utah, Salt Lake City, Utah, USA

${ }^{4}$ Division of Preventive Medicine, Department of Family and Preventive Medicine, University of California, San Diego, California, USA

${ }^{5}$ Department of Clinical Sciences, University of Texas Southwestern Medical Center, Dallas, Texas, USA

${ }^{6}$ Department of Biobehavioral Health, Pennsylvania State University, University Park, Pennsylvania, USA

Correspondence to

Dr John M Ruiz;

johnruiz@email.arizona.edu

\section{ABSTRACT}

Introduction Psychosocial factors are increasingly recognised as important determinants of cardiovascular disease risk. The North Texas Heart Study aims to understand the mechanisms responsible for this association with a focus on social vigilance (ie, scanning the environment for social threats). There is also growing interest in supplementing traditional methods (eg, survey assessment of psychosocial risk paired with crosssectional and longitudinal health outcomes) with daily or repeated momentary assessment of psychosocial factors. However, there are relatively few longitudinal studies directly comparing these approaches with hard endpoints. Methods and analysis The North Texas Heart Study proposes a longitudinal measurement burst design to examine psychosocial determinants of subclinical atherosclerosis. A sample of 300 healthy community participants, stratified by age and gender, will complete survey measures, as well as 2 days of ecological momentary assessment at baseline and at a 2-year follow-up. A range of psychosocial and behavioural factors, objective biomarkers, as well as carotid intima-media thickness (CIMT) will be assessed at both time points. Unadjusted and adjusted models will evaluate crosssectional associations and determinants of change in the CIMT.

Ethics and dissemination The Institutional Review Board at the study coordinating institute (University of North Texas) has approved this study. Positive, negative or inconclusive primary and ancillary findings will be disseminated in scientific journals and conferences.

\section{INTRODUCTION}

Substantial evidence supports psychosocial stress as an important risk factor for the development of cardiovascular disease (CVD) morbidity and mortality, as well as all-cause mortality. ${ }^{1-7}$ For example, more frequent exposure to social stress, including interpersonal conflict, discrimination, hostile work environments and disadvantaged neighbourhoods increase the risk for coronary heart disease (CHD) morbidity and mortality. ${ }^{8-14}$ Individual differences in the tendency to

\section{Strengths and limitations of this study}

- This study is significant in that it evaluates social vigilance as a novel though ecologically valid behavioural risk factor for stress-related disease.

- The mixed methods longitudinal design facilitates both a broad relationship understanding of social vigilance and its relationship to atherosclerotic disease as well as daily biobehavioural interactions that may mediate disease progression.

- The multimethod assessment of social vigilance including use of the same measure in traditional survey, daily diary and momentary assessment formats will allow comparison of granularity differences in association with outcomes.

- Despite our best efforts, it is possible that either the amount of measureable disease will be too low to detect in our healthy sample or that insufficient change will occur within the 2-year follow-up to ascertain vigilance effects on progression.

- It is also possible that despite conceptual distinctions, social vigilance simply does not translate into unique disease impact.

perceive or experience social stress as more severe has also been prospectively associated with accelerated atherosclerotic progression, adverse cardiac events and mortality. ${ }^{15-19}$

Vigilance reflects a sensory intake process ${ }^{20}$ where an environment or potential threat is continually monitored and reappraised in order to detect any change in status. Social vigilance is one behaviour that could potentially link stress and a broad array of individual, social and contextual moderators to CVD (see figure 1). Social vigilance refers to the act of monitoring the social environment for potential interpersonal challenges or threats, as well as monitoring change in status of a perceived threat. $^{21} 22$ This behaviour may be automatic, as in general surveillance, or effortful when monitoring a specific target or environment. Notably, several factors may evoke more 


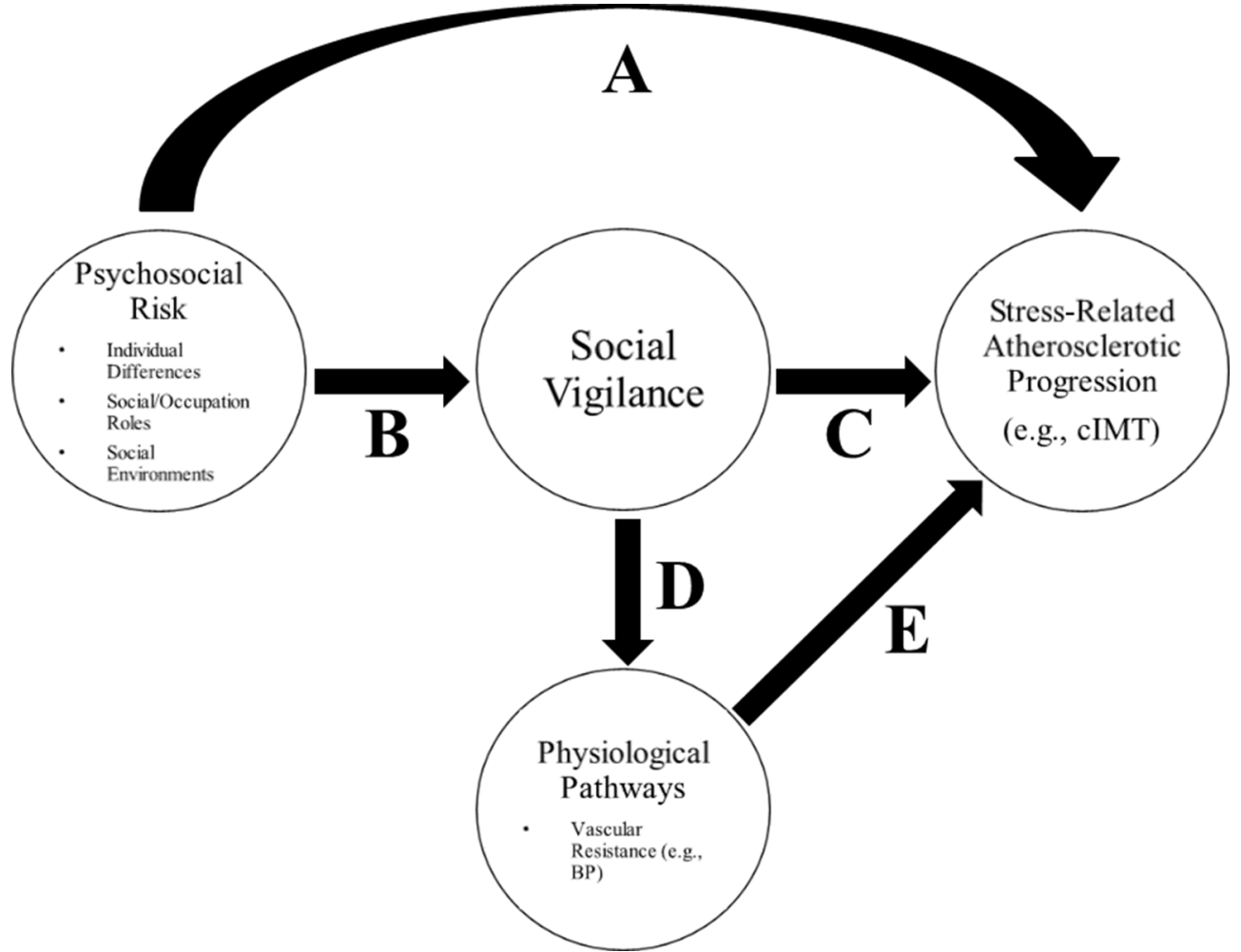

Figure 1 Conceptual model illustrating the role of social vigilance. Path A demonstrates the broad relationship between psychosocial stress and atherosclerotic disease. Paths $\mathrm{B}$ and $\mathrm{C}$ represent the hypothesised mediational role of social vigilance. Path $\mathrm{D}$ represents the acute physiological concomitants of socially vigilant behaviour. Path $\mathrm{E}$ represents the direct physiological pathway to disease as a function of chronicity. BP, blood pressure; cIMT, carotid intima-media thickness.

chronic vigilance of the social environment and may include individual differences (eg, hostility and negative affectivity), more challenging social environments (eg, unsafe neighbourhoods and hostile work environments) and social roles requiring vigilant observation (eg, security work and caregiving). Although social vigilance may be adaptive in some contexts (eg, dangerous situations), sustained vigilance or hypervigilance may have important health consequences.

Prior evidence suggests that vigilant behavior evokes a pattern of vascular resistance characterized by increases in blood pressure (BP) and total peripheral resistance (TPR), often with little change in heart rate (HR). ${ }^{23} 24$ Such patterns may represent a significant risk factor for CVD. ${ }^{125}$ This finding is supported by a number of traditional tasks that aim to evoke vigilant behaviour, including mirror tracing, ${ }^{26-28}$ the Stroop colour-word interference $\operatorname{task}^{2629}$ and computerised signal detection tasks. ${ }^{30}$ Additional evidence from our lab broadens these findings to vigilance of social stimuli. ${ }^{24}$ For example, men that viewed a video of a potential competitor exhibited larger increases in both BP and TPR compared with those who monitored a non-competitive social target. Together, this research supports a mediating pathway linking psychological stress to increased physical disease risk through increased vascular resistance.

Experience sampling, or ecological momentary assessment (EMA), is a research method used to assess psychosocial factors in temporal proximity to their experience with the inference that this approach improves the ecological validity of the experience relative to more traditional survey recall efforts. It is superior to traditional self-report measures for assessing the frequency, nature and content of acute experiences as it samples in real time and in naturalistic settings. ${ }^{31-33}$ This method, in combination with assessment of emotional, psychophysiological and biological processes in real time, may provide important information regarding the contexts that increase risk and potentially reveal mechanisms that may underlie physical change. For example, a series of research studies by Kamarck and colleagues demonstrated that daily task demands were cross-sectionally associated with carotid intima-media thickness (IMT) ${ }^{34}$ and longitudinally predictive of disease progression, with additional evidence suggesting potential mediation by baseline ambulatory BP. ${ }^{35}$ The application of this evolving real-time microprocess assessment methodology should help to improve our understanding of how concepts like vigilance are experienced in everyday life to influence disease risk and progression.

\section{AIMS}

The North Texas Heart Study is a longitudinal investigation that will examine the impact of social vigilance and daily experiences on the development of subclinical atherosclerosis. The first aim of this study is to examine social vigilance as a predictor of progression of carotid 
IMT in an initially healthy community sample. Second, this study aims to examine whether ambulatory BP mediates the relationship between social vigilance and carotid IMT. To help elucidate the mechanisms that underlie or serve to promote atherosclerotic progression, an exploratory aim of this study is to capture measures of psychosocial well-being, health behaviours, emotional responses, subjective and objective sleep quantity and quality, and inflammatory biomarkers at study intake and again at a 2-year follow-up. It is hypothesised that greater social vigilance will be associated with higher carotid IMT values at each time point, as well as predict carotid IMT progression over time. We also expect that daily BP responses to momentary social vigilance will mediate the effects of vigilance on carotid IMT progression at 2-year follow-up. Findings from this study will help to elucidate the behaviourally manifested CVD risk factors, which can then serve as intervention targets to reduce CVD risk.

\section{METHODS AND ANALYSIS \\ Project overview}

This longitudinal investigation proposes to evaluate social vigilance as a cognitive, behavioural and emotional risk factor for higher levels of atherosclerosis in a racially and economically diverse sample recruited from Denton County, Texas, USA. The study will employ multiple methods for data collection including self-report surveys, daily experience assessments, ambulatory monitoring, medical examinations, imaging and biomarker analysis. Data collection will take place at study entry and again at a 2-year follow-up. This multimethod approach will provide rich data affording the opportunity to examine psychosocial contributions to objective disease progression and contrast the predictive value of variations in sampling from survey to momentary experiences.

\section{Participants and recruitment}

A baseline community sample of 300 adults ( 150 men and 150 women), ages 21-70 years will be recruited from Denton County, Texas. The sample will be stratified by age (by decades) within gender and race/ethnicity in order to examine age-related effects. Participants will be recruited through a variety of sources including, but not limited to, advertisements in local newspapers, flyers, community and university websites, hospital postings and community organisation postings.

Denton County is an economically and racially diverse community located approximately 30 miles north of Dallas. The Denton County population was estimated to be 658616 persons in 2009 with a racial make-up described as $83.3 \%$ white, $8.3 \%$ black and $5.7 \%$ Asian. In addition, $17.9 \%$ of the community is of Hispanic/ Latino ethnicity including a sizeable immigrant population. The current study will aim to oversample Hispanic/ Latinos and non-Hispanic blacks to achieve at least 25\% Hispanic/Latinos and 15\% non-Hispanic black/African Americans with approximately $54.5 \%$ non-Hispanic white

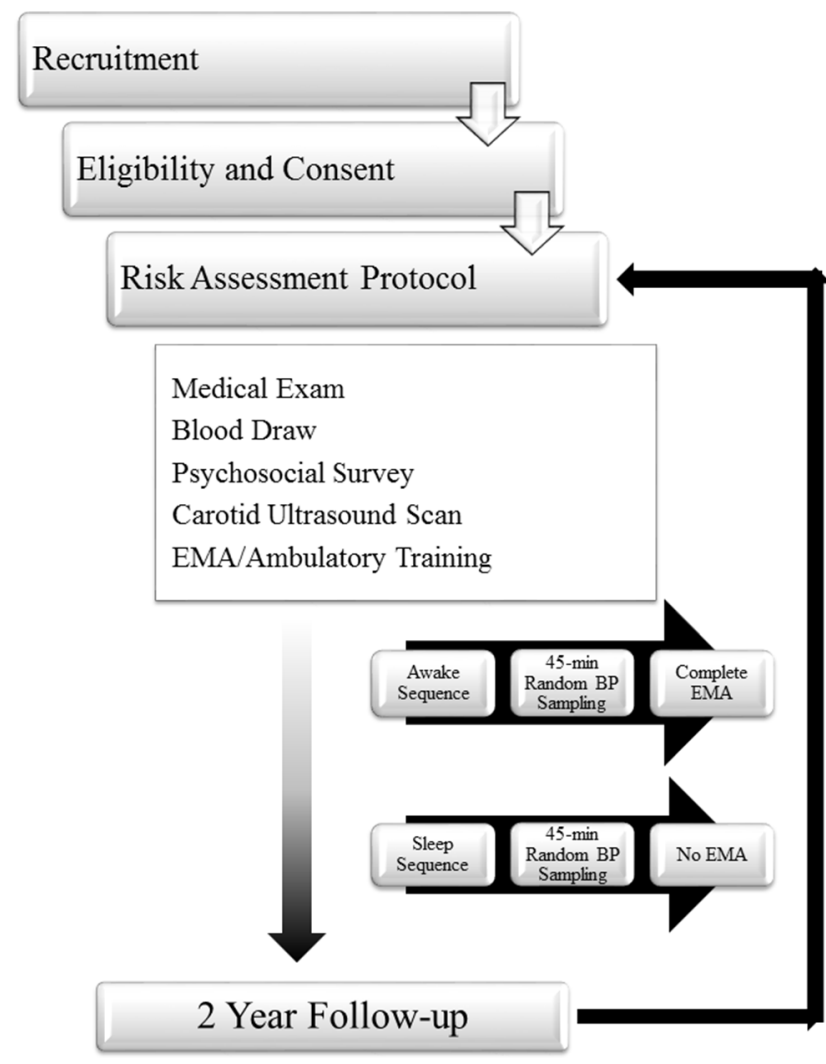

Figure 2 The North Texas Heart Study protocol. BP, blood pressure; EMA, ecological momentary assessment.

and 5.5\% non-Hispanic Asian. To achieve our racial/ ethnic minority representation goals, we will partner with neighbourhood/community leaders to promote the study aims and benefits to participants. Consistent with this effort, we will also use promotores de salud (Hispanic/ Latino community health workers, patient navigators or healthcare advocates) to bolster recruitment of Hispanic/ Latino participants. Given that promotores come from the communities they aim to serve, they represent a unique cultural and linguistic pathway that may also facilitate recruitment for research.

To be eligible, participants must be: (1) over 21 years of age, (2) residing within Denton County and (3) have written and verbal fluency in English language. Exclusion criteria are: (1) cognitive impairment (ie, dementia), (2) previous history of myocardial infarction or tertiary cardiac interventions (eg, coronary artery bypass surgery and implanted cardiac defibrillator), (3) pregnancy within last year or anticipating pregnancy during study period and (4) an occupation that requires shift work. Screening will be conducted to verify no evidence of manifest cardiac disease. The use of anti-inflammatory and lipid management medications will be assessed as covariates but will not be a cause for exclusion.

\section{Procedure}

The study protocol is outlined in figure 2 . The study will be coordinated by a research team at the University of 
North Texas. All laboratory visits will be conducted at a single vascular medicine clinic located in the community.

\section{Screening and consent}

Potential participants will be screened for eligibility by the study coordinator via phone. A target matrix of gender, age by decade and race/ethnicity will be used to ensure the sampling goals: equal gender and racial/ ethnic representation within each decade from 21 to 70 years at time of enrolment. Participants that meet recruitment targets, as well as inclusion/exclusion criteria, will be scheduled for a session.

All sessions will take place on Thursday mornings between the hours of 08:00 and 10:00. Participants will be instructed to fast overnight ( 8 hours). This constrained appointment time (all participants run in the same 2- to 3-hour morning block) is important to ensure the fidelity of inflammatory biomarkers. ${ }^{36}$ Participants will meet the study coordinator at the clinic at their scheduled appointment time to provide written, informed consent and begin the protocol.

\section{Laboratory visit}

After consent is provided, participants will undergo a brief physical exam that includes a review of systems (ROS), personal and family medical history, current medications and health conditions, health behaviours and detailed cardiac disease history. A fasting blood draw to assess lipids and inflammatory markers will be performed. A vascular technologist will then perform a complete bilateral ultrasound imaging study of the extracranial carotid arteries and related vasculature. Finally, participants will complete a questionnaire package that includes measures of vigilance, social experiences, personality and health behaviours. Prior to leaving the laboratory, all participants will be fitted with a wrist-worn Actiwatch Spectrum (Phillips-Respironics) sleep/wake activity monitor, an ambulatory blood pressure monitor (ABPM; Oscar II; Suntech), and will be given a preprogrammed mobile device for a 2-day/2night ambulatory/EMA assessment.

\section{Ambulatory/EMA assessment}

ABPM measurements will be programmed to assess BP at random times within $45 \mathrm{~min}$ intervals throughout the day. This random sampling procedure prevents participants from anticipating a measurement and hence altering their activities. Participants will be instructed to complete the EMA protocol using the provided mobile device in response to each BP measurement during awake hours. Participants will be further instructed to complete two additional EMA-based surveys: (1) an End-of-Day (EOD) Survey completed at bedtime and (2) a morning survey on awakening (ie, Wake Survey). The Actiwatch Spectrum will be programmed to assess activity at $30 \mathrm{~s}$ epochs, and participants will be instructed to leave the watch on for the entire 2-day/2-night monitoring period. Participants will be instructed to turn off the ABPM and remove it at bedtime on the first night and to attach and activate it on awakening the next morning. On the second night, participants will wear the ABPM in order to collect nocturnal BP data (eg, in order to estimate nocturnal dipping). On the third day, participants will meet the study coordinator at the clinic to return the ambulatory/EMA equipment. Participants will also be asked about the development of acute illness symptoms since the previous appointment as a means to account for potential prodromal illness states (ie, asymptomatic states of infection) on inflammation markers.

\section{Longitudinal assessment}

Longitudinal change on all measures will be evaluated by repeating the protocol at a 2-year follow-up visit using the same procedure outlined above. Specifically, participants will repeat the medical exam, questionnaire package, fasting blood draw, ultrasound scan and 2-day/2-night ambulatory/EMA protocol. A follow-up period of 2 years was deemed an appropriate follow-up length based on previous research that demonstrated the impact of psychosocial risk factors on change in carotid IMT over this time period. ${ }^{37-40}$

\section{Measures}

Three types of measures will be collected: (1) psychosocial survey data (table 1), (2) ecological sampling data including both EMA and daily diaries (table 2) and (3) ambulatory physiological and clinical outcomes data (table 3). Below we detail specific domains and constructs.

\section{Demographics}

Demographic data including age, race, ethnicity, household income, education attainment, marital status, household size, occupational status (Hollingshead classification), ${ }^{41}{ }^{42}$ typical work hours and military experience will be assessed through self-report. These variables will be used as covariates in models testing the first aim and may also serve ancillary purposes at a later time.

\section{Psychosocial well-being and health behaviours}

A self-report questionnaire package will be used to collect four domains of conceptually relevant psychosocial risk moderators (see table 1 for additional detail). Domains include: (1) individual-level factors including health behaviours, mood/affect and personality traits, (2) social factors, (3) social roles and (4) contextual factors. Measures were chosen based on four criteria: (1) relevance to the vigilance construct, (2) relevance to disease risk, (3) psychometric properties and (4) length.

\section{Social vigilance}

Social vigilance will be assessed with a new instrumentthe Social Vigilance Questionnaire (SVQ). The SVQ is a newly developed 10-item, three-factor self-report measure assessing the degree to which a person engages in stress-related vigilance or monitoring of the social environment. Participants will be provided the stem, 'In social 
Table 1 Domains, constructs and measures in the psychosocial survey

Domain Construct Measure Description

Health behaviours

Physical activity Global Physical Activity Questionnaire ${ }^{71}$

\begin{tabular}{|c|c|c|}
\hline Smoking & Tobacco use* & $\begin{array}{l}\text { Assesses current and past smoking and nicotine use } \\
\text { behaviour }\end{array}$ \\
\hline Alcohol use & Alcohol Use Questionnaire* & $\begin{array}{l}\text { Assesses current and past alcohol use, including frequency } \\
\text { and amount }\end{array}$ \\
\hline Sleep & Insomnia Severity Index ${ }^{72}$ & $\begin{array}{l}\text { Assesses the perceived severity of DSM-V chronic } \\
\text { insomnia symptoms }\end{array}$ \\
\hline & $\begin{array}{l}\text { Snoring, Tired, Observed, } \\
\text { Blood Pressure }^{73}\end{array}$ & $\begin{array}{l}\text { Assesses self-reported symptoms of sleep apnoea; } \\
\text { score } \geq 2 \text { indicative of apnoea risk }\end{array}$ \\
\hline & Self-Assessment of Sleep* & $\begin{array}{l}\text { Assesses sleep quantity and quality during the week and } \\
\text { weekends, number of hours needed to feel rested, overall } \\
\text { sleep quality and duration of current sleep pattern }\end{array}$ \\
\hline
\end{tabular}

Psychosocial stress

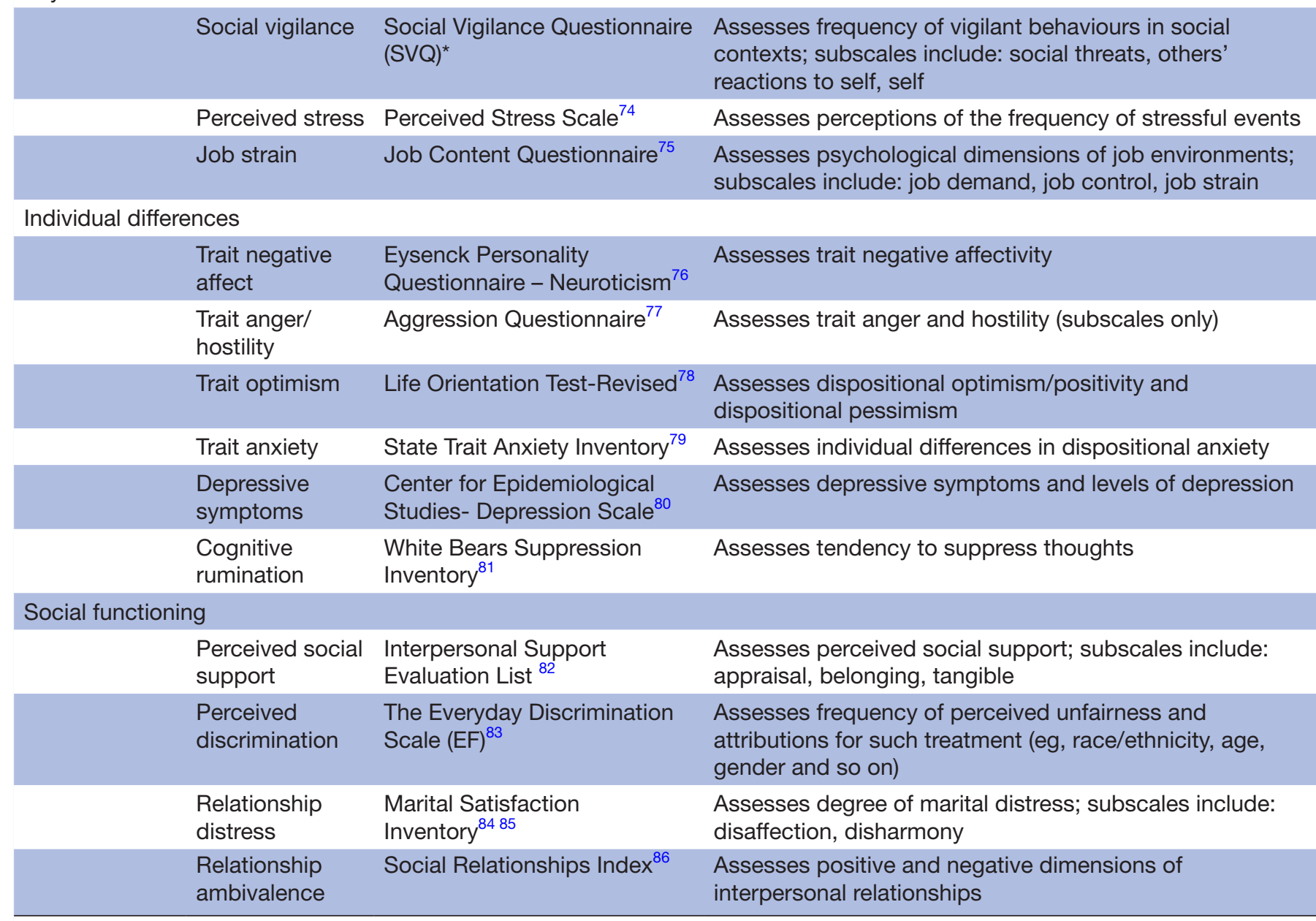

DSM-V, Diagnostic and Statistical Manual of Mental Disorders, Fifth Edition * measure developed for this study

situations...' followed by the items that are rated using a 4-point Likert scale ranging from 0 'almost never' to 4 'almost always'.

We propose to assess the frequency of socially vigilant behaviour using the SVQ with two conceptually related but distinct methodologies. First, the SVQ will be
Assesses physical activity at work, during transportation and during leisure; used to calculate metabolic equivalents Assesses current and past smoking and nicotine use behaviour and amount

sleea; Assesses sleep quantity and quality during the week and weekends, number of hours needed to feel rested, overall

Assesses frequency of vigilant behaviours in socia contexts; subscales include: social threats, others' reactions to self, self Assesses psychological dimensions of job environments Assesses trait negative affectivity

Assesses trait anger and hostility (subscales only)

Assesses dispositional optimism/positivity and dispositional pessimism

Assesses individual differences in dispositional anxiety

Assesses tendency to suppress thoughts

Assesses perceived social support; subscales include: appraisal, belonging, tangible

Assesses frequency of perceived unfairness and attributions for such treatment (eg, race/ethnicity, age, gender and so on)

Assesses degree of marital distress; subscales include: disaffection, disharmony interpersonal relationships included in the self-report questionnaire package that all participants complete at baseline. The second approach will be to assess the frequency of vigilant behaviour in daily life using EMA sampling. The full 10-item SVQ will be adapted to the EMA format by altering the stem to sample vigilant behaviour 'since the last reading' and will 
Table 2 Constructs and items assessed using EMA Cuff Survey, End-of-Day Survey and Wake Survey

\begin{tabular}{lll}
\hline Assessment & Construct & Description \\
\hline EMA Cuff-Survey & Physical attributes & Seven items assessing physical covariates at cuff inflation: \\
& Posture \\
& Activity level \\
& Talking during measurement \\
& Temperature \\
& Location \\
& Recent consumption of food, alcohol, nicotine \\
& Recent exercise
\end{tabular}

Affect

12 items (1 'not at all' to 7 'extremely'):

Energetic (lively and energetic)

Relaxed (relaxed and calm)

Anxious (nervous and tense)

Sad (sad and depressed)

Positive (cheerful and happy)

Hostile (hostile and angry)

\begin{tabular}{|c|c|c|}
\hline & Stress & $\begin{array}{l}\text { Three items assessing: } \\
\text { Stressful event since prior measure (yes/no) } \\
\text { Stressor severity ( } 1 \text { 'not at all' to } 7 \text { 'extremely') } \\
\text { General stress levels since previous inflation } \\
\text { (1 'not at all' to } 7 \text { 'extremely') }\end{array}$ \\
\hline & Social exposure & One item assessing social exposure (yes/no) \\
\hline & Social vigilance & $\begin{array}{l}\text { If social exposure=yes: } \\
\text { 10-item Social Vigilance Questionnaire (SVQ) }\end{array}$ \\
\hline & Interpersonal exposure & One item assessing interactions (yes/no) \\
\hline & Interpersonal interactions & $\begin{array}{l}\text { If interpersonal exposure=yes, three items assessing: } \\
\text { Identify the relationship (who was interaction with) } \\
\text { Rate the positive quality ( } 1 \text { 'not at all' to } 7 \text { 'extremely') } \\
\text { Rate the negative quality ( } 1 \text { 'not at all' to } 7 \text { 'extremely') } \\
\text { If interpersonal exposure=no, three items assessing: } \\
\text { Reason for no interaction (eg, no opportunities and avoiding) } \\
\text { Likelihood of avoiding an interaction by the next cuff inflation } \\
\text { (1 'not at all' to } 7 \text { 'extremely') } \\
\text { Likelihood of initiating an interaction by the next cuff inflation } \\
\text { ( } 1 \text { 'not at all' to } 7 \text { 'extremely') }\end{array}$ \\
\hline & State optimism & $\begin{array}{l}\text { Two items assessing positive and negative outlook in next } 30 \mathrm{~min} \\
\text { (1 'strongly disagree' to } 7 \text { 'strongly agree') }\end{array}$ \\
\hline End-of-Day Survey & $\begin{array}{l}\text { Daily summary of affect, stress, } \\
\text { social vigilance }\end{array}$ & $\begin{array}{l}\text { Stem - 'In general today...' } \\
12 \text { items assessing affect ( } 1 \text { 'not at all' to } 7 \text { 'extremely'): } \\
\text { Energetic (lively and energetic) } \\
\text { Relaxed (relaxed and calm) } \\
\text { Anxious (nervous and tense) } \\
\text { Sad (sad and depressed) } \\
\text { Positive (cheerful and happy) } \\
\text { Hostile (hostile and angry) } \\
\text { Two items assessing stress: } \\
\text { How stressed did you feel today (1 'not at all' to } 7 \text { 'extremely') } \\
\text { How did stress compare to typical day ( } 1 \text { 'much more' to } 7 \text { 'far below') } \\
\text { 10-item SVQ }\end{array}$ \\
\hline
\end{tabular}

EMA, ecological momentary assessment. 
Table 3 Ambulatory physiological and clinical outcomes

\begin{tabular}{|c|c|c|}
\hline Assessment & Construct & Description \\
\hline $\begin{array}{l}\text { Ambulatory blood } \\
\text { pressure (ABP) }\end{array}$ & Blood pressure & $\begin{array}{l}\text { Oscillometric data capture of blood pressure at random intervals within } \\
45 \text { min blocks over } 2 \text { days and } 1 \text { night to obtain measures of: } \\
\text { Systolic blood pressure } \\
\text { Diastolic blood pressure } \\
\text { Mean arterial stiffness } \\
\text { Heart rate }\end{array}$ \\
\hline \multirow[t]{3}{*}{ Fasting blood draws } & Metabolic & Fasting blood glucose \\
\hline & Lipids & $\begin{array}{l}\text { Low-density lipoprotein } \\
\text { High-density lipoprotein } \\
\text { Triglycerides } \\
\text { Total cholesterol }\end{array}$ \\
\hline & Inflammation & $\begin{array}{l}\text { High-sensitivity C-reactive protein } \\
\text { Tumour necrosis factor-alpha } \\
\text { Interleukin-6 }\end{array}$ \\
\hline B-Mode ultrasound & Carotid artery imaging & $\begin{array}{l}\text { Bilateral assessment of the carotid vasculature using standard angles }\left(90^{\circ} \text {, }\right. \\
\left.150^{\circ}, 210^{\circ}, 270^{\circ}\right) \text { to obtain the following derived measures: } \\
\text { Mean max far common carotid artery } \\
\text { Mean max bifurcation (BIF) } \\
\text { Mean max internal carotid artery (ICA) } \\
\text { Mean max combined BIF/ICA }\end{array}$ \\
\hline
\end{tabular}

be assessed at each BP measurement if a social interaction has taken place. This method will allow for a dynamic measure of vigilant behaviour at $\sim 45$ min intervals during the day.

\section{Medical history and exam}

At the laboratory session, a registered nurse will meet with participants to document health at study initiation and describe their personal and family health history. The physical exam includes assessment of standard anthropomorphic measures (eg, height, weight, waist circumference and so on), health behaviour information (eg, smoking, alcohol use, physical activity, diet and sleep) and an ROS (eg, functioning and symptoms in all major regulatory system including but not limited to cardiovascular, respiratory, ears, nose, mouth, throat, gastrointestinal and so on). Three measures of resting BP will be collected and aggregated as a baseline measure of clinic BP. A phlebotomist will perform a fasting blood draw to assess a baseline lipid profile (ie, total cholesterol, high-density lipoprotein, low-density lipoprotein and triglycerides), fasting glucose, and to collect serum to assess inflammatory markers.

\section{Carotid imaging}

The extracranial carotid arteries will be imaged using B-mode ultrasound. The approach yields a two-dimensional (2D) image of the carotid vasculature enabling measurement of wall thickness. Advances in ultrasound imaging using Doppler allow for measuring and visualising blood flow velocity. For the current study, all participants will undergo a carotid artery duplex scan. The term 'duplex' refers to the combination of B-mode with Doppler, which creates a 2D image with blood flow. This approach allows for traditional carotid IMT measurement, as well as information on blood flow direction, flow velocities, occlusive disease, significant plaque and other abnormalities. ${ }^{43} 44$

All scans will be performed by experienced sonographers. Briefly, Dicom images will be captured for the common (CCA), bifurcation (BIF) and the internal carotid artery (ICA) segments interrogated from four standard angles $\left(90^{\circ}, 150^{\circ}, 210^{\circ}\right.$ and $\left.270^{\circ}\right)$. Consistent with recommendations, ${ }^{45-47}$ a Meijer's Carotid Arc (Meijer Medical Ultrasound; Voorschoten, The Netherlands) will be used to improve the precision of measurement points and to improve reliability of within-participant measurement over time. 
Using Vascular Research Tools, V.5.0 (Medical Imaging Applications, Coralville, Iowa, USA), two readers blinded to the characteristics of the participants will score the carotid IMT offline. This software uses a semiautomated edge detection algorithm to ascertain the thickness of the intima-media complex in the designated region of interest (ROI) for each frame in the series of the clip. Consistent with the literature, carotid IMT is defined as the distance between the intimal-luminal and the medial-adventitial interfaces of the arterial segment within the ROI. Each segment will also be analysed for the presence or absence of plaques. Plaques will be given a quality score using the following criteria: (1) size (ie, small, medium and large), (2) heterogeneity/homogeneity and (3) fibrous, fatty and calcified. The software generates average, minimum and maximum IMT scores for each segment at each angle. The maximum IMT value will be used as the marker of focus in accordance with prior recommendations ${ }^{48}$ and the team's past work. ${ }^{49}{ }^{50}$ Mean of the maximums (mean of the maximum scores for each of the four angels for a given segment) will be derived for the CCA, BIF and ICA.

\section{Inflammatory biomarkers}

To examine inflammation, as it pertains to potential effects of social vigilance on atherosclerosis, we will assess high sensitivity C-reactive protein (CRP), tumour necrosis factor-alpha (TNF- $\alpha$ ) and interleukin 6 (IL-6). These markers are hypothesised to represent different points along the inflammatory process from stress to atherosclerosis, and their inclusion allows us to address not only presence of inflammation but also address how active inflammatory processes may influence all phases of the atherosclerotic processes. ${ }^{51-53}$ CRP has received the greatest attention as a marker of inflammatory processes and is a robust predictor of future cardiovascular risk in a number of prospective studies. ${ }^{54-58}$ However, CRP is a relatively late step in the inflammatory process and understanding earlier cytokine patterns that precede it and initiate the inflammatory cascade is important for theoretical models linking psychosocial factors relationships to health outcomes. To this point, TNF- $\alpha$ is generally conceptualised as an acute phase cytokine and IL-6 is conceptualised as a central early inflammatory cytokine that can directly initiate and modulate the inflammatory cascade. ${ }^{5960}$

\section{Ambulatory BP}

Participants will be fitted with an ABPM (Oscar 2; Suntech Medical Instruments, Raleigh, North Carolina) to wear during the day and evening to capture measures of systolic blood pressure, diastolic blood pressure and HR. The Oscar 2 was designed specifically for ambulatory assessments and is the only ABPM clinically validated to all three international standards. ${ }^{61} 62$ The sensors and the cuff are unobtrusively worn under the participants' clothing, and only a small control unit (approximately $4.7 \times 2.8 \times 1.2$ inches; $284 \mathrm{~g}$ ) attached to the participant's belt is partially exposed. The ABPM will be programmed to assess $\mathrm{BP}$ at random times during $45 \mathrm{~min}$ intervals during the first and second study days and the second study night (2 days, 1 night). Data will be cleaned using established criteria. ${ }^{63}$ Participants will be trained how to remove and put back on the unit after bathing or vigorous exercise but otherwise will be asked to keep the monitor on as instructed.

\section{Ecological momentary assessment}

Participants will be provided with a preprogrammed mobile device that serves as the EMA delivery platform. Daily experiences will be assessed using three surveys: (1) the EMA/Cuff Survey, which serves as the primary momentary sampling survey throughout the study day, (2) the EOD Survey to assess participant's summary impressions of their day and (3) the Wake Survey, which will take the form of a sleep diary by assessing aspects of sleep quantity and quality (table 2). Programming includes drop down menus and slide bars for Likert scales.

\section{The EMA/Cuff Survey}

The EMA/Cuff Survey will serve as the primary daily sampling survey and will be paired with ABPM, where the BP sampling will serve as the triggering event for the participant to complete the survey. The EMA/Cuff Survey is divided into six measurement sections: physical parameters, affect, stress, social exposure, interpersonal interactions and perceived outlook (see table 2 for additional detail). Importantly, this survey is balanced such that participants receive relatively equal numbers of questions (range 38-40 questions) regardless of responses. This reduces the likelihood of inadvertently entraining certain answers that are associated with shorter survey requirements.

\section{EOD Survey}

Participants will be instructed to complete the EOD Survey at bedtime on the first and second days. The purpose is to assess summary ratings of: affect, stress and vigilance during that day.

\section{The Wake Survey}

Participants will be instructed to complete the Wake Survey when they wake on the second and third study days. The Wake Survey is a retrospective sleep diary ${ }^{64}$ that assesses aspects of the previous night's sleep. It is comprised of items that will provide measures of: sleep onset latency, time in bed, number of awakenings, wake after sleep onset, terminal wakefulness, total sleep time, sleep efficiency, sleep quality and use of sleep medication.

\section{Sleep assessment}

\section{Subjective sleep quantity and quality}

Participant sleep quantity and quality will be assessed during each laboratory visit using three self-report measures (table 1). Overall, these measures are designed to measure: (1) self-reported sleep duration and quality, (2) subjective insomnia symptom severity and (3) self-reported sleep apnoea risk. 


\section{Objective sleep quantity and quality}

The Actiwatch Spectrum Actiwatches (Philips-Respironics, Bend, Oregon) are compact, wrist-worn, battery-operated activity monitors with physical characteristics similar to a small wristwatch. The monitor utilises a motion sensor known as an 'accelerometer' to monitor the occurrence and degree of motion. This type of sensor integrates the degree and speed of motion and produces a small signal where magnitude and duration depend on the amount of motion. Activity data will be collected over $30 \mathrm{~s}$ epochs. In addition, Actiwatch Spectrum has an on-board event marker button that the patient presses to identify bedtime and out of bed times. This information is analysed with Actiware (Philips-Respironics) software using settings of medium activity threshold and 10 immobile minutes to detect wakefulness. Derived variables include: bedtime, wake time, time in bed, sleep onset latency, number of awakenings during the night, wake time after sleep onset, total sleep time and sleep efficiency, using proprietary scoring algorithms in the software. Actigraphy and sleep diary/EMA data will be combined to optimise assessment of sleep parameters.

\section{Sample size and power analysis}

Sample size and power were estimated from previous research examining conceptually relevant individual difference measures and carotid IMT. These data suggest effect sizes were comparable across a range of trait negative affects including hostility, anger and anxiety. ${ }^{38} 406566$ Assuming that the correlation between the change in social vigilance score and the change in carotid IMT is at least 0.20 , a total of 210 subjects who complete baseline and follow-up will yield $83 \%$ power to detect $20 \%$ correlation against the null hypothesis of no correlation using a two-sided test with a significance level of 0.05. Borrowing from Stewart and colleagues, ${ }^{38}$ power is boosted to $90 \%$ if we assume that there is an increasing trend in carotid IMT over tertiles of social vigilance assuming that a common SD within each tertile is 0.10 . Finally, in Stewart and colleagues ${ }^{38}$ study, 360 out of 464 enrolled subjects completed baseline and follow-up assessments of carotid IMT. Assuming a similar completion rate (ie, $78 \%$ ), we need to recruit a total of 270 subjects to account for dropout. Hence, our targeted sample of 300 participants should be sufficient to detect the hypothesised relationships accounting for progression rate, strength of association and attrition.

\section{ETHICS AND DISSEMINATION}

The aim of this study is to determine whether social vigilance may serve as a trigger for the pathway linking stress to atherosclerotic disease risk, and whether BP reactivity during daily life mediates this risk. We expect that greater social vigilance (ie, higher self-report and more frequent daily experience) will be associated with higher carotid IMT scores at each time point and predict faster rate of progression over time and that momentary BP reactivity will (at least in part) mediate these effects. This study is significant because it takes the critical next step of evaluating social vigilance as a predictor of objective disease. It will also provide evidence for the mechanistic pathways underlying the relationship. Identification of CVD-relevant biobehavioural pathways can inform targeted interventions to modify such behaviour.

One of the unique contributions afforded by the proposed design and methodology will be the ability to demonstrate the benefits of variation in sampling frequency of psychosocial factors in relation to physical health biomarkers and processes. As explained, three gradients of assessment of psychosocial constructs will be employed in this study-traditional survey measures, daily summary measures and EMA. This provides for convergent validity across time scales and methods, but also allows for the development and testing of multilevel and multisystem hypotheses, determining the unique and synergistic influences of social vigilance, and other opportunities that arise from this rich and layered data. For example, prior research in pain suggests that global self-report measures and EMA are complementary approaches, each explaining unique variance in outcomes. ${ }^{67} 68$ More recent work has suggested that EMA data may be more sensitive than global retrospective reports in predicting health changes, including carotid IMT. ${ }^{35} 6970$ Thus, we will be able to explore the predictive and clinical utility of social vigilance assessed at different levels and time scales and by using different methods.

The proposed study is also innovative in its approach to examining the biobehavioural pathway between psychosocial constructs, such as vigilance, and physical health indicators, such as atherosclerosis. Whereas examination of blood-based biomarkers such as inflammation are routinely used to link psychosocial factors to atherosclerosis, we pair EMA with ABMP in addition to inflammatory markers. This sampling approach allows us to test hypotheses regarding acute physiological reactions to vigilance in daily life as a mediator of the stress-related progression in atherosclerosis with intervention implications.

\section{LIMITATIONS}

Despite our best efforts, we recognise that there are several challenges to the proposed study. First, it is possible that either the amount of measureable disease will be too low to detect in our sample or that insufficient change will occur within the 2-year follow-up to detect vigilance effects on progression. To address this possibility, we have chosen to have all participants undergo a complete bilateral duplex ultrasound to ascertain disease information including plaque formations and blood flow in all extracranial vessels. This approach essentially has a power effect commensurate with increasing the number of sampling data points. By imaging the entire vasculature, we increase the observational space in which to detect disease relative to more 
focused investigations of specific vascular segments while maintaining our ability to examine those specific regions.

A second possibility is that vigilance may be more influential at different time points in the disease process. For example, social vigilance may generally produce mild to moderate cardiovascular reactions that are below threshold for disease development but may be detrimental in the context of existing disease. This possibility would require a completely different approach and involve examining the effects of vigilance in a clinical sample; we leave this for future studies.

A third possibility is that vigilant behaviour varies as a function of age and stage of life. For example, working-age individuals may be higher in social vigilance as a function of more frequent social interactions through work or school. Older individuals including retirees may engage in less vigilance as a result of advancing age, disease and reduced mobility effects on ability and desire to seek out social engagement. We intend to include age in our models, but it is possible that we are underpowered to detect significant, age-related cohort effects.

Finally, it is possible that despite conceptual distinctions, social vigilance simply may not translate into unique disease impact. A strength of our approach is the inclusion of conceptually related variables and the ability to test this possibility. Although we expect that vigilance will emerge as a significant moderator of carotid IMT, the alternative outcome would also be an important contribution to the literature given the widespread hypothesising of vigilance effects on disease and the lack of objective data.

This study protocol is approved by the Institutional Review Board at the University of North Texas (coordinating location) and will abide by all regulations aimed at valuing and preserving respect and safety in human factors research. Study findings will be disseminated in scientific journals and conferences.

\section{CONCLUSION}

We believe that this study represents an innovative and significant conceptual advance in understanding the relationship between social vigilance and atherosclerotic risk and a methodological demonstration of the benefits of variations in candidate psychosocial risk factor sampling for testing relationships with physical health endpoints. This will be the first investigation to examine social vigilance as a determinant of objectively measured atherosclerosis in humans. Importantly, this evidence will be a necessary step in demonstrating that vigilance is distinct from other psychosocial risk factors, such as neuroticism and hostility, in that it is a basic downstream mechanism by which such individual differences influence CHD. Findings will improve understanding of the basic biobehavioural pathways linking social stress to CHD risk, demonstrate the methodological utility of varying sampling techniques and inform targeted interventions to ameliorate stress-related disease.
Contributors JMR, DJT, BNU, TWS, MA, CA and JMS developed the protocol with each leading a specific assessment section. DJT leads the sleep assessment; BNU leads the assessment of blood-based biomarkers and ambulatory blood pressure; TWS contributed to the conceptualisation and measurement of stress; MA leads the measurement and scoring of carotid intima media thickness; CA leads the analytic strategy; JMS leads the EMA assessment; and JMR leads conceptualisation and measurement of social vigilance and serves as the overall principal investigator. The manuscript was drafted by all as well as additional writing and editing by JJ. All authors have given their approval for the to be published.

Funding This research was generouslysupported by grant number R01HL109340 from the National Heart, Lung, and Bloodlnstitute (NHLBI) and the Basic Behavioral and Social Sciences Research0pportunity Network (OppNet), National Institutes of Health (PI: John M. Ruiz).

Competing interests None declared.

Patient consent No-this study is conducted in the United States (Texas) and will not report individual results. This paper is a protocol paper and discusses the rationale and design of the proposed study.

Ethics approval University of North Texas, Denton, TX, USA.

Provenance and peer review Not commissioned; peer reviewed for ethical and funding approval prior to submission.

Data sharing statement Researchers interested in testing hypotheses with the data are encouraged to contact the corresponding author and complete a data proposal form for review.

Open Access This is an 0pen Access article distributed in accordance with the Creative Commons Attribution Non Commercial (CC BY-NC 4.0) license, which permits others to distribute, remix, adapt, build upon this work non-commercially, and license their derivative works on different terms, provided the original work is properly cited and the use is non-commercial. See: http://creativecommons.org/ licenses/by-nc/4.0/

Author note The authors would like to thank Dr. CarlosP Cruz, Sheila Van Bree, James Garcia, Jessica Dietch, Lacy Fenn, MichaelDaniels, Denton Vascular Lab, Minor Emergency of Denton, and the Denton, Texascommunity for their support of this work.

(c) Article author(s) (or their employer(s) unless otherwise stated in the text of the article) 2017. All rights reserved. No commercial use is permitted unless otherwise expressly granted.

\section{REFERENCES}

1. Richardson S, Shaffer JA, Falzon L, et al. Meta-analysis of perceived stress and its association with incident coronary heart disease. $A m J$ Cardiol 2012;110:1711-6.

2. Kaplan JR, Manuck SB, Clarkson TB, et al. Social stress and atherosclerosis in normocholesterolemic monkeys. Science 1983;220:733-5.

3. Jennings JR, Kamarck TW, Everson-Rose SA, et al. Exaggerated blood pressure responses during mental stress are prospectively related to enhanced carotid atherosclerosis in middle-aged finnish men. Circulation 2004;110:2198-203.

4. Yusuf S, Hawken S, Ounpuu S, et al. Effect of potentially modifiable risk factors associated with myocardial infarction in 52 countries (the INTERHEART study): case-control study. Lancet 2004;364:937-52.

5. Lazzarino Al, Hamer M, Stamatakis E, et al. The combined association of psychological distress and socioeconomic status with all-cause mortality: a national cohort study. JAMA Intern Med 2013;173:22-7.

6. Russ TC, Stamatakis E, Hamer M, et al. Association between psychological distress and mortality: individual participant pooled analysis of 10 prospective cohort studies. BMJ 2012;345:e4933.

7. Iso H, Date C, Yamamoto A, et al. Perceived mental stress and mortality from cardiovascular disease among japanese men and women: the Japan Collaborative Cohort Study for evaluation of Cancer risk sponsored by Monbusho (JACC Study). Circulation 2002;106:1229-36.

8. Smith TW, Ruiz JM. Psychosocial influences on the development and course of coronary heart disease: current status and implications for research and practice. J Consult Clin Psychol 2002;70:548-68.

9. Schoenthaler AM, Schwartz J, Cassells A, et al. Daily interpersonal conflict predicts masked hypertension in an urban sample. Am J Hypertens 2010;23:1082-8. 
10. Tomfohr L, Cooper DC, Mills PJ, et al. Everyday discrimination and nocturnal blood pressure dipping in black and white americans. Psychosom Med 2010;72:266-72.

11. Kivimäki M, Nyberg ST, Batty GD, et al. Job strain as a risk factor for coronary heart disease: a collaborative meta-analysis of individual participant data. Lancet 2012;380:1491-7.

12. Kivimäki M, Virtanen M, Elovainio $M$, et al. Work stress in the etiology of coronary heart disease--a meta-analysis. Scand J Work Environ Health 2006;32:431-42.

13. Borrell LN, Diez Roux AV, Rose K, et al. Neighbourhood characteristics and mortality in the Atherosclerosis risk in Communities Study. Int J Epidemiol 2004;33:398-407.

14. Lynch J, Kaplan GA, Salonen R, et al. Socioeconomic status and progression of carotid atherosclerosis. prospective evidence from the Kuopio ischemic Heart Disease risk factor study. Arterioscler Thromb Vasc Biol 1997;17:513-9.

15. Kubzansky LD, Kawachi I, Spiro A, et al. Is worrying bad for your heart? A prospective study of worry and coronary heart disease in the normative aging study. Circulation 1997;95:818-24.

16. Mathur R, Pérez-Pinar M, Foguet-Boreu Q, et al. Risk of incident cardiovascular events amongst individuals with anxiety and depression: a prospective cohort study in the east London primary care database. J Affect Disord 2016;206:41-7.

17. Newman JD, Davidson KW, Shaffer JA, et al. Observed hostility and the risk of incident ischemic heart disease: a prospective population study from the 1995 canadian Nova Scotia health Survey. J Am Coll Cardiol 2011;58:1222-8.

18. Wong JM, Sin NL, Whooley MA. A comparison of cook-medley hostility subscales and mortality in patients with coronary heart disease: data from the heart and soul study. Psychosom Med 2014:76:311-7.

19. Chida Y, Steptoe A. The association of anger and hostility with future coronary heart disease: a meta-analytic review of prospective evidence. J Am Coll Cardiol 2009;53:936-46.

20. Lazarus RS, Folkman S. Stress, appraisal, and coping. New York, NY Springer, 1984:456.

21. Schneiderman N, McCabe PM. Psychophysiologic strategies in laboratory research. Schneiderman SM N, ed. Weiss, \& P. G. Kaufmann. New York: Plenum: Handbook of research methods in cardiovascular behavioral medicine, 1989:157-200.

22. Williams RB , . Patterns of reactivity and stress. In: Matthews KA Detre T, Dembroski TM, Falkner SMW, Manuck SB, Williams RB, eds Handbook of stress, reactivity, and cardiovascular disease. New York: Wiley, 1986:p. 109-26.

23. Gump BB, Matthews KA. Vigilance and cardiovascular reactivity to subsequent stressors in men: a preliminary study. Health Psychol 1998;17:93-6.

24. Smith TW, Ruiz JM, Uchino BN. Vigilance, active coping, and cardiovascular reactivity during social interaction in young men. Health Psychol 2000;19:382-92.

25. Panaite V, Salomon K, Jin A, et al. Cardiovascular recovery from psychological and physiological challenge and risk for adverse cardiovascular outcomes and all-cause mortality. Psychosom Med 2015;77:215-26.

26. Steptoe A, Kivimäki M, Lowe G, et al. Blood pressure and fibrinogen responses to mental stress as predictors of Incident hypertension over an 8-Year period. Ann Behav Med 2016;50:898-906

27. Roemmich JN, Feda DM, Seelbinder AM, et al. Stress-induced cardiovascular reactivity and atherogenesis in adolescents. Atherosclerosis 2011;215:465-70.

28. Saab PG, Llabre MM, Hurwitz BE, et al. Myocardial and peripheral vascular responses to behavioral challenges and their stability in black and white Americans. Psychophysiology 1992;29:384-97.

29. Boutcher YN, Boutcher SH. Cardiovascular response to Stroop: effect of verbal response and task difficulty. Biol Psychol 2006;73:235-41.

30. Gendolla GH, Richter M. Cardiovascular reactivity during performance under social observation: the moderating role of task difficulty. Int J Psychophysiol 2006;62:185-92.

31. Stone A, Shiffman S, Atienza A, et al. The science of Real-Time Data Capture: self-reports in Health Research. New York: Oxford University Press, 2007.

32. Mehl MR, Robbins ML. Naturalistic observation sampling: the Electronically Activated Recorder (EAR). Handbook of Research methods for studying Daily Life. New York: Guilford Press, 2012:176-92.

33. Smyth JM, Smyth JM, Stone A. Ecological Momentary Assessment Research in Behavioral medicine. J Happiness Stud 2003;4:35-52.

34. Kamarck TW, Muldoon MF, Shiffman S, et al. Experiences of demand and control in daily life as correlates of subclinical carotid atherosclerosis in a healthy older sample. Health Psychol 2004;23:24-32.

35. Kamarck TW, Shiffman S, Sutton-Tyrrell K, et al. Daily psychological demands are associated with 6-year progression of carotid artery atherosclerosis: the Pittsburgh healthy Heart Project. Psychosom Med 2012;74:432-9.

36. O'Connor MF, Bower JE, Cho HJ, et al. To assess, to control, to exclude: effects of biobehavioral factors on circulating inflammatory markers. Brain Behav Immun 2009;23:887-97.

37. Räikkönen K, Matthews KA, Sutton-Tyrrell K, et al. Trait anger and the metabolic syndrome predict progression of carotid atherosclerosis in healthy middle-aged women. Psychosom Med 2004;66:903-8.

38. Stewart JC, Janicki DL, Muldoon MF, et al. Negative emotions and 3-year progression of subclinical atherosclerosis. Arch Gen Psychiatry 2007;64:225-33.

39. Janssen I, Powell LH, Matthews KA, et al. Depressive symptoms are related to progression of coronary calcium in midlife women: the study of Women's Health Across the Nation (SWAN) Heart Study. Am Heart J 2011;161:1186-91.

40. Elovainio M, Keltikangas-Järvinen L, Kivimäki M, et al. Depressive symptoms and carotid artery intima-media thickness in young adults: the Cardiovascular risk in Young Finns Study. Psychosom Med 2005;67:561-7.

41. Hauser R, Warren JR. Socioeconomic indexes for occupations: a review, update, and critique. Center for Demography and Ecology: University of Wisconsin-Madison, 1996.

42. Stevens G, Featherman DL. A revised socioeconomic index of occupational status. Soc Sci Res 1981;10:364-95.

43. Ehler D, Carney DK, Dempsey AL, et al. Guidelines for cardiac sonographer education: recommendations of the American Society of Echocardiography Sonographer training and Education Committee. J Am Soc Echocardiogr 2001;14:77-84.

44. LeFevre ML; U.S. Preventive Services Task Force. Screening for asymptomatic carotid artery Stenosis: U.S. Preventive Services Task Force recommendation statement. Ann Intern Med 2014;161:356-62.

45. Dogan S, Duivenvoorden R, Grobbee DE, et al. Ultrasound protocols to measure carotid intima-media thickness in trials; comparison of reproducibility, rate of progression, and effect of intervention in subjects with familial hypercholesterolemia and subjects with mixed dyslipidemia. Ann Med 2010;42:447-64.

46. Dogan S, Plantinga Y, Crouse JR, et al. Algorithms to measure carotid intima-media thickness in trials: a comparison of reproducibility, rate of progression and treatment effect. $J$ Hypertens 2011;29:2181-93.

47. Stein JH, Korcarz CE, Hurst RT, et al. American Society of Echocardiography Carotid Intima-Media Thickness Task Force. Use of carotid ultrasound to identify subclinical vascular disease and evaluate cardiovascular disease risk: a consensus statement from the American Society of Echocardiography Carotid IntimaMedia Thickness Task Force. Endorsed by the Society for Vascular Medicine. J Am Soc Echocardiogr 2008;21:93-111.

48. Allan PL, Mowbray PI, Lee AJ, et al. Relationship between carotid intima-media thickness and symptomatic and asymptomatic peripheral arterial disease. The Edinburgh Artery Study. Stroke 1997:28:348-53.

49. Allison MA, Laughlin GA, Barrett-Connor E. Association between the ankle-brachial index and carotid intimal medial thickness in the Rancho Bernardo Study. Am J Cardiol 2006;98:1105-9.

50. Roepke SK, Allison M, Von Känel R, et al. Relationship between chronic stress and carotid intima-media thickness (IMT) in elderly Alzheimer's disease caregivers. Stress 2012;15:121-9.

51. Ross R. Atherosclerosis--an inflammatory disease. N Engl J Med 1999;340:S419-S420

52. Ross R. Atherosclerosis is an inflammatory disease. Am Heart $J$ 1999;138(5 Pt 2):S419-S420.

53. Libby P. Inflammation in atherosclerosis. Nature 2002;420:868-74.

54. Libby P, Ridker PM. Inflammation and atherosclerosis: role of C-reactive protein in risk assessment. Am J Med 2004;116(Suppl $6 \mathrm{~A}): 9 \mathrm{~s}-16$.

55. Pizzi C, Costa GM, Santarella L, et al. Depression symptoms and the progression of carotid intima-media thickness: a 5-year follow-up study. Atherosclerosis 2014;233:530-6.

56. Hashimoto $\mathrm{H}$, Kitagawa $\mathrm{K}$, Hougaku $\mathrm{H}$, et al. C-reactive protein is an independent predictor of the rate of increase in early carotid atherosclerosis. Circulation 2001;104:63-7.

57. Paul A, Ko KW, Li L, et al. C-reactive protein accelerates the progression of atherosclerosis in apolipoprotein E-deficient mice. Circulation 2004:109:647-55.

58. van Dijk EJ, Prins ND, Vermeer SE, et al. C-reactive protein and cerebral small-vessel disease: the Rotterdam scan Study. Circulation 2005;112:900-5. 
59. Kiecolt-Glaser JK, McGuire L, Robles TF, et al. Emotions, morbidity, and mortality: new perspectives from psychoneuroimmunology. Annu Rev Psychol 2002;53:83-107.

60. Rieckmann P, Tuscano JM, Kehrl JH. Tumor necrosis factor-alpha (TNF-alpha) and interleukin-6 (IL-6) in B-lymphocyte function. Methods 1997;11:128-32.

61. Goodwin J, Bilous M, Winship S, et al. Validation of the Oscar 2 oscillometric 24-h ambulatory blood pressure monitor according to the british hypertension Society protocol. Blood Press Monit 2007;12:113-7.

62. Jones SC, Bilous M, Winship S, et al. Validation of the OSCAR 2 oscillometric 24-hour ambulatory blood pressure monitor according to the International Protocol for the validation of blood pressure measuring devices. Blood Press Monit 2004;9:219-23.

63. Marler MR, Jacob RG, Lehoczky JP, et al. The statistical analysis of treatment effects in 24-hour ambulatory blood pressure recordings. Stat Med 1988;7:697-716.

64. Carney CE, Buysse DJ, Ancoli-Israel S, et al. The consensus sleep diary: standardizing prospective sleep self-monitoring. Sleep 2012;35:287-302.

65. Matthews KA, Owens JF, Kuller LH, et al. Are hostility and anxiety associated with carotid atherosclerosis in healthy postmenopausal women? Psychosom Med 1998:60:633-8.

66. Matthews KA, Räikkönen K, Sutton-Tyrrell K, et al. Optimistic attitudes protect against progression of carotid atherosclerosis in healthy middle-aged women. Psychosom Med 2004;66:640-4.

67. Stone AA, Broderick JE, Porter LS, et al. The experience of rheumatoid arthritis pain and fatigue: examining momentary reports and correlates over one week. Arthritis Care Res 1997;10:185-93.

68. Kikuchi H, Yoshiuchi K, Miyasaka N, et al. Reliability of recalled self-report on headache intensity: investigation using ecological momentary assessment technique. Cephalalgia 2006;26:1335-43.

69. John-Henderson NA, Kamarck TW, Muldoon MF, et al. Early Life Family Conflict, Social interactions, and carotid artery Intima-Media Thickness in Adulthood. Psychosom Med 2016;78:319-26.

70. Joseph NT, Kamarck TW, Muldoon MF, et al. Daily marital interaction quality and carotid artery intima-medial thickness in healthy middleaged adults. Psychosom Med 2014;76:347-54.

71. Organization WH. Global physical activity questionnaire (GPAQ). Geneva, Switzerland 1975.

72. Morin CM. Insomnia: psychological Assessment and Management. New York: The Guilford Press, 1993.
73. Chung F, Yegneswaran B, Liao P, et al. STOP questionnaire: a tool to screen patients for obstructive sleep apnea. Anesthesiology 2008;108:812-21.

74. Cohen S, Kamarck T, Mermelstein R. A global measure of perceived stress. J Health Soc Behav 1983;24:385-96.

75. Karasek R, Brisson C, Kawakami N, et al. The Job Content Questionnaire (JCQ): an instrument for internationally comparative assessments of psychosocial job characteristics. J Occup Health Psychol 1998;3:322-55.

76. Eysenck HJ, Eysenck SBG. Manual of the Eysenck Personality Questionnaire. London: Hodder \& Stoughton, 1975.

77. Buss AH, Perry M. The aggression questionnaire. J Pers Soc Psychol 1992;63:452-9.

78. Scheier MF, Carver CS, Bridges MW. Distinguishing optimism from neuroticism (and trait anxiety, self-mastery, and self-esteem): a reevaluation of the Life Orientation Test. J Pers Soc Psychol 1994;67:1063-78.

79. Spielberger CD, Gorssuch RL, Lushene PR, et al. Manual for the state trait anxiety inventory. Palo Alto: Consulting Psychologists Press, Inc, 1983.

80. Radloff LS. The CES-D Scale: a Self-Report Depression Scale for Research in the General Population. Appl Psychol Meas 1977;1:385-401.

81. Wegner DM, Zanakos S. Chronic thought suppression. J Pers 1994;62:615-40.

82. Cohen S, Mermelstein R, Kamarck T, et al; Measuring the functional components of social support. In: Sarason G, Sarason BR, eds. Social support: theory, research, and application. the Hague: martinus Nijhoff, 1985:p. 73-94.

83. Williams DR, Yan Yu, Jackson JS, et al. Racial differences in physical and Mental Health: socio-economic Status, stress and discrimination. J Health Psychol 1997;2:335-51.

84. Snyder DK. Marital satisfaction Inventory-Revised (MSI-R) manual. Los Angeles: Western Psychological Services, 1997.

85. Herrington RL, Mitchell AE, Castellani AM, et al. Assessing disharmony and disaffection in intimate relationships: revision of the marital satisfaction inventory factor scales. Psychol Assess 2008;20:341-50.

86. Uchino BN, Holt-Lunstad J, Uno D, et al. Heterogeneity in the social networks of young and older adults: prediction of mental health and cardiovascular reactivity during acute stress. J Behav Med 2001;24:361-82. 